Review

\title{
Colony Stimulating Factor-1 and its Receptor in Gastrointestinal Malignant Tumors
}

\author{
Hong-wu Li, Shi-lei Tang \\ General Surgery Department, The Fourth Affiliated Hospital of China Medical University, Shenyang, Liaoning Province, China, 110032. \\ $\square$ Corresponding author: Dr. Shi-lei Tang, General Surgery Department, The Fourth Affiliated Hospital of China Medical University, Chongshan East Road \\ No.4, Huanggu District, Shenyang, Liaoning Province, China, 110032. E-mail: sltang@cmu.edu.cn. \\ () The author(s). This is an open access article distributed under the terms of the Creative Commons Attribution License (https://creativecommons.org/licenses/by/4.0/). \\ See http://ivyspring.com/terms for full terms and conditions.
}

Received: 2021.03.12; Accepted: 2021.10.01; Published: 2021.10.17

\begin{abstract}
Gastrointestinal malignant tumor is the fourth most common cancer in the world and the second cause of cancer death. Due to the susceptibility to lymphatic metastasis and liver metastasis, the prognosis of advanced tumor patients is still poor till now. With the development of tumor molecular biology, the tumor microenvironment and the cytokines, which are closely related to the proliferation, infiltration and metastasis, have become a research hotspot in life sciences. Colony stimulating factor-1 (CSF-1), a polypeptide chain cytokine, and its receptor CSF-1R are reported to play important roles in regulating tumor-associated macrophages in tumor microenvironment and participating in the occurrence and development in diversities of cancers. Targeted inhibition of the CSF-1/CSF-1R signal axis has broad application prospects in cancer immunotherapy. Here, we reviewed the biological characters of CSF-1/CSF-IR and their relationship with gastrointestinal malignancies.
\end{abstract}

Key words: colony stimulating factor-1; colony stimulating factor-1 receptor; tumor-associated macrophages; gastrointestinal malignant tumors

\section{Introduction}

Malignant tumors of the gastrointestinal (GI) tract are one of the common malignant tumors in China, among which gastric cancer is the fourth most common cancer in the world and the second cause of cancer death $[1,2]$. On account of a high frequent occurrence of lymphatic and liver metastasis, advanced GI malignant tumors lead to a poor prognosis and a serious threat to the life and living quality of patients [3-5]. With the development of tumor molecular biology and related disciplines, tumor microenvironment and the involved cytokines, which are closely related to the occurrence, proliferation, infiltration and metastasis of GI malignant tumors, have become a research hotspot in life sciences [6]. Understanding the tumor microenvironment (TME) and its role in tumor treatment is the key step in the development of more effective treatment methods [7]. Colony stimulating factor-1 (CSF-1), a polypeptide chain cytokine, has been found that when combined with colony stimulating factor-1 receptor (CSF-1R), it could mobilize a variety of bone marrow precursor cells, promote cell proliferation, differentiation and migration, and enhance the function of mature granulocytes [8-10]. CSF-1R is mainly expressed on the surface of hematopoietic stem cells, myeloid progenitor cells, and mature granulocytes [11, 12]. Recent studies have found that CSF-1 in the tumor microenvironment could combine with its receptor CSF-1R in liver cancer, ureteral cancer, pancreatic cancer, gastric cancer, intestinal cancer and other malignant tumors [7, 13-17], and participate in biological processes such as promoting tumor cell proliferation, inhibiting tumor cell apoptosis and inducing blood vessel formation, etc. Targeted inhibition of the CSF-1/CSF-1R signal axis has broad application prospects in cancer immunotherapy [18-20]. Therefore, it is important to explore the CSF-1 and CSF-1R signaling pathways related to tumorassociated macrophages (TAM) and their specific mechanisms in the comprehensive prevention and treatment of gastrointestinal malignancies. Here, we 
summarized the biological functions of CSF-1/ CSF-1R and reviewed cancer promoting mechanisms regulated by CSF-1/CSF-1R axis in neoplasms, particularly in gastrointestinal tract malignant tumors.

\section{Characteristics, structure and function of CSF-1 and its receptor CSF-1R}

\section{Characteristics, structure and function of CSF-1}

CSF-1 is an important cytokine in vivo and a member of the hematopoietic growth factor family. It plays an important role in different stages of hematopoiesis [21]. CSF-1 could directly act on CSF-1R, usually expressed in platelets, hematopoietic stem cells, bone marrow progenitor cells and mature bone marrow cells, leading to the acceleration of the formation of granulocyte colonies, the differentiation and proliferation of bone marrow cells, the enhancement of the function of mature neutrophils, and the acceleration of cell migration [22]. In the study of hematopoietic cells in vitro, CSF-1 can stimulate different hematopoietic stem cells to form cells in semisolid media and stimulate bone marrow and spleen macrophage cell lines [23]. In recent years, studies have shown that CSF-1 is highly expressed in many malignant tumors, which can accelerate invasion, proliferation and metastasis and induce the formation of tumor vessels by binding with CSF-1R $[16,19,24]$. The high expression of CSF-1 can also be observed in tissues obtained from surgery, suggesting that high expression of CSF-1 may be an indicator of the prognosis of patients [25].

CSF-1 is a glycoprotein composed of 174 amino acid residues [26]. The full length of the CSF-1 gene is $2.5 \mathrm{~kb}$, including 5 exons, 4 introns, 5 cysteines, and two disulfide bonds, which are formed between Cys-36 and -42 and Cys-64 and -74. Disulfide bonds are an important factor in maintaining the biological function of CSF-1 [27, 28]. Naturally formed CSF-1 is linked by four single rings through amino acids. The CSF content in the healthy state is low. When inflammation occurs in the body, the concentration of CSF in plasma increases significantly $[29,30]$.

\section{General characteristics, structure and function of CSF-1R}

CSF-1R belongs to the receptor tyrosine kinase signaling system. One of the characters of such a receptor is that when the ligand binds to the receptor recognition sites outside the cell membrane, the receptor will then aggregate and phosphorylate the tyrosine kinase-activated receptors within the cell membrane, finally leading to the phosphorylation of the tyrosine residues of effector proteins and the alteration of the biological activity of the effector [31]. A recent study has demonstrated that in primary colorectal cancer, the elevated expression of receptor tyrosine kinase CSF1R at the tumor invasion was associated with poor patient survival and a mesenchymal-like subtype [24]. Effector proteins include many factors related to cell proliferation and differentiation and many other components of signal-mediated systems. An obvious characteristic of this system is that in addition to rapid reaction time, it can also exert a long-term effect on cells [18]. Since most factors that regulate cell proliferation and differentiation work in this way, thus this receptor is closely related to the occurrence and development of tumors.

CSF-1R, also known as FMS kinase, is the coding product of the c-fms proto-oncogene [32]. C-fms is located at 5q33-3 of the human chromosome. Human CSF-1R synthesizes a $130 \mathrm{kDa}$ immature transmembrane glycoprotein (gp130c-fms) from the rough endoplasmic reticulum, which is then modified by $\mathrm{N}$-terminal oligosaccharide chains to form a mature $150 \mathrm{kDa}$ glycoprotein (gp150c-fms) during intracellular transport. CSF-1R is secreted from the Golgi to the cytoplasm and then localized to the cell membrane. It can be converted from gp130c-fms to gp150c-fms within 1 hour [33]. Human CSF-1R is a 972-amino acid polypeptide, consisting of four main parts: a 19 amino acid signal peptide sequence, a 493 amino acid ligand recognition and binding sequence outside membrane, a 25 amino acid transmembrane segment, and a 435 amino acid C-terminal tyrosine kinase catalytic sequence [34]. CSF-1R is very similar in structure with platelet-derived growth factor (PDGF) receptor and belongs to the type III receptor tyrosine kinase family. The extracellular domain consists of five immunoglobulin-like rings for ligand binding. The intracellular domain contains tyrosine residues for self-phosphorylation, the kinase catalytic region contains three tyrosine self-phosphorylation sites $(699,708,723)$, and the C-terminal region also has a tyrosine residue (809) for self-phosphorylation [35]. After binding with CSF-1R, a ligand-receptor complex is formed, which is then endocytosed by cells and degraded in lysosomes. This is referred to as the internalization of CSF-1R [36]. The extracellular signal produced by the binding of the receptor to its ligand is transmitted and amplified to the cells, which leads to the activation of tyrosine kinase and the phosphorylation of the receptor itself, thus initiating and stimulating the signal transduction pathway of cell proliferation. Therefore, the activation of the receptor tyrosine kinase is the first step for CSF-1R to play its role [37]. 
When membrane receptors are abnormally activated, tyrosine residues can be phosphorylated to induce ligand conformational changes that stimulate continuous activation of tyrosine kinases, leading to cell growth and proliferation and eventually carcinogenesis [38]. Choudlhury et al. reported that an abnormal increase in the activity of the CSF-1R tyrosine kinase domain can activate the proto-oncogene c-raf- 1 , thus increasing the activity of serine/threonine kinases, which encode the product of CSF-1R. This can enhance or accelerate the transduction of intracellular growth stimuli, resulting in the amplification effect on the biological function of CSF-1R [39]. Macrophage colony-stimulating factor binds to CSF-1R and activates its tyrosine kinase, which plays an important role in the survival, proliferation, differentiation and embryonic development of monocyte macrophage lines [13]. Overexpression of CSF-1R and its ligands causes abnormal activation of the cell signaling pathway mediated by CSF-1R and participates in the processes of tumorigenesis and inflammation. Similar studies have shown that CSF-1R and its ligand CSF-1 can activate the receptor tyrosine kinase signaling system and continuously increase the activity of tyrosine kinase, thus changing the biological activity of the effector proteins. This allows the corresponding cells to continue growing and proliferating, which play a long-term role in the cell, and finally develop into cancer [16]. Therefore, inhibitors targeting CSF-1R kinase can inhibit receptor phosphorylation and block the receptor-mediated cell signaling pathway, which is a potential target drug for the treatment of malignant tumors and inflammatory diseases [40].

\section{The relationship between CSF-1/CSF-1R axis and TAM in malignant tumors}

Recent studies have shown that TAM is closely related to macrophage colony stimulating factor (M-CSF) [41]. Macrophages that reside in the tumor microenvironment are called tumor-associated macrophages. They are the main inflammatory immune cells in the tumor microenvironment and are involved in tumor immunosuppression, angiogenesis, invasion, and metastasis [42, 43].

The interaction between CSF- 1 and CSF-1R could cause receptor dimerization, tyrosine phosphorylation, and the subsequent interaction with multiple intracellular signaling pathways such as Ras, MAPK, PI-3K, JAK, finally producing various biological effects [44]. CSF-1, as a tumor molecular marker, are highly expressed in a variety of tumors. Over-expression of CSF-1 or CSF-1R is associated with tumor aggressiveness and poor prognosis [45, 46]. Recently, it has been found that the application of inhibitors can block the CSF-1R receptor and significantly reduce the invasiveness and proliferation of endometrial cancer, of which the progression could be promoted by TAM [47]. CSF-1 secreted by endometrial cancer cells promotes the migration and proliferation of macrophages. The results show that the interaction between CSF-1 and its receptor plays an important role in promoting macrophage infiltration and endometrial cancer progression [48].

According to their phenotypes and secreted cytokines, TAM is divided into two types of polarization: classically activated M1 type and selectively activated M2 type macrophages [49, 50]. M1 type macrophages mainly secrete pro-inflammatory factors and exert host immune functions against microbial inflammation and killing tumor cells. M2 type macrophages play a local anti-inflammatory effect in the later stage of inflammation, promote wound repair and fibrosis, participate in the formation of tumor stroma, and promote tumor growth, metastasis, and tumor angiogenesis [51]. Macrophage polarization typing is the body's need for the diversity of immune function, which is related to the microenvironment and disease state of macrophages. Various pathological products or factors in vivo and in vitro become an important inducement for the macrophage polarization. Abnormally high expression of CSF-1 is related to pathological processes such as tumors and inflammation $[52,53]$. Due to the different functions of M1 and M2 macrophages, there may be mutual conversion between these two types of cells. Blocking the CSF-1/CSF-1R signaling pathway interferes with tumor progression by regulating TAM, reducing tumor invasion and proliferation [54].

Previous studies have demonstrated that CSF-1 and interleukin 34 (IL-34) signal via CSF-1R play important roles in macrophage differentiation in several inflammatory and oncological preclinical models $[55,56]$. For example, Blockade of both CSF-1 and IL-34 is protective in murine models of colitis and ileitis [55]. However, numerous recent studies have found that CSF-1/CSF-1R axis blockade can improve the efficiency of immune checkpoint inhibitors, especially PD-L1. Thus, in terms of tumor control, the combination therapy targeting CSF-1/CSF-1R axis and immune checkpoint molecular has more reliable efficacy $[57,58]$. CSF-1/CSF-1R signaling mediates tumor-associated macrophages recruitment and M2 polarization. In experimental mesotheliomas, combined a highly selective small molecule CSF-1R inhibitor-BLZ945 with an anti-PDL1 agent was more effective in retarding tumor growth compared to each monotherapy [58]. Moreover, AMG 820, an antiCSF-1R antibody, showed acceptable safety profile in 
combination with pembrolizumab in adults with advanced solid tumors by reducing CSF-1 dependent CD16 expressing monocytes, and increasing PD-L1 expression and infiltrating T-lymphocyte numbers in advanced solid tumor biopsies [57].

Alternative ligands of CSF-1R, including IL-34 [59], have been discovered, but most macrophages require signaling via the CSF1-CSF-1R axis. Except for macrophage, CSF-1/CSF-1R axis can promote tumor progression by interacting with other cells in tumor microenvironment, for instance, CAFs [60]. In addition to fibroblasts, CSF-1 can be secreted by tumor cells, suggesting that it may play a pro-tumorigenic role (Fig. 1). Consistent with this, in metastatic PDAC, tumor-cell-derived CSF-1 induces macrophages to produce granulin, a secreted glycoprotein that promotes fibroblast activation and spurs tumor growth [61].

The CSF-1/CSF-1R signaling pathway modulates the production, differentiation, and function of TAMs. However, the discovery of selective CSF-1R inhibitors devoid of type III kinase activity has proven to be challenging in tumor treatment. Barbara Czako et al. discovered a potent, highly selective, and orally bioavailable CSF1R inhibitor, IACS-9439, which was proposed as a potential therapy to reduce TAMs, especially the protumor, immune-suppressive M2 TAMs and promote macrophage polarization toward the M1 phenotype by targeting CSF-1R [62]. Recently, immunotherapy has gradually become the focus of cancer treatment. However, the majority of patients with "cold" tumors do not benefit from immunotherapy [63]. Interestingly, a targeted delivery strategy, which modified cell-penetrating TAT peptide by using CSF-1R inhibitor, successfully activate immune response through blocking the CSF-1/CSF-1R pathway and reducing M2 macrophages and thus promoting anti-tumor effector CD8+T-lymphocyte infiltration in "cold" colon cancer [64].

\section{Expression and significance of CSF-1 and CSF-1R in gastrointestinal malignant tumors}

CSF-1 is a hematopoietic growth factor that acts through the cfms/CSF-1R. The CSF-1/CSF-1R axis is considered to be involved in the invasion and development of various types of cancer [65, 66]. Studies have reported that elevated expression of CSF-1/CSF-1R significantly correlated with disease progression and with a poor overall survival (OS) and disease-free survival (DFS) of patients with gastric cancer. Furthermore, the high co-expression of CSF-1 and CSF-1R was an independent prognostic factor for OS, DFS, lymph node and peritoneal metastasis, indicating that the CSF-1/CSF-1R axis may be a clinically useful prognostic and predictive biomarker for lymph node and peritoneal metastasis and a potential therapeutic target in gastric cancer [16]. Other studies have found that CSF is a key factor to drive CXCL8 secretion in M2 type TAM. The high expression of CXCL8 is significantly associated with decreased $\mathrm{CD}^{+}$and $\mathrm{Ki}^{+} 7^{+} \mathrm{T}$ cells infiltration and unfavorable clinical outcome in gastric cancer
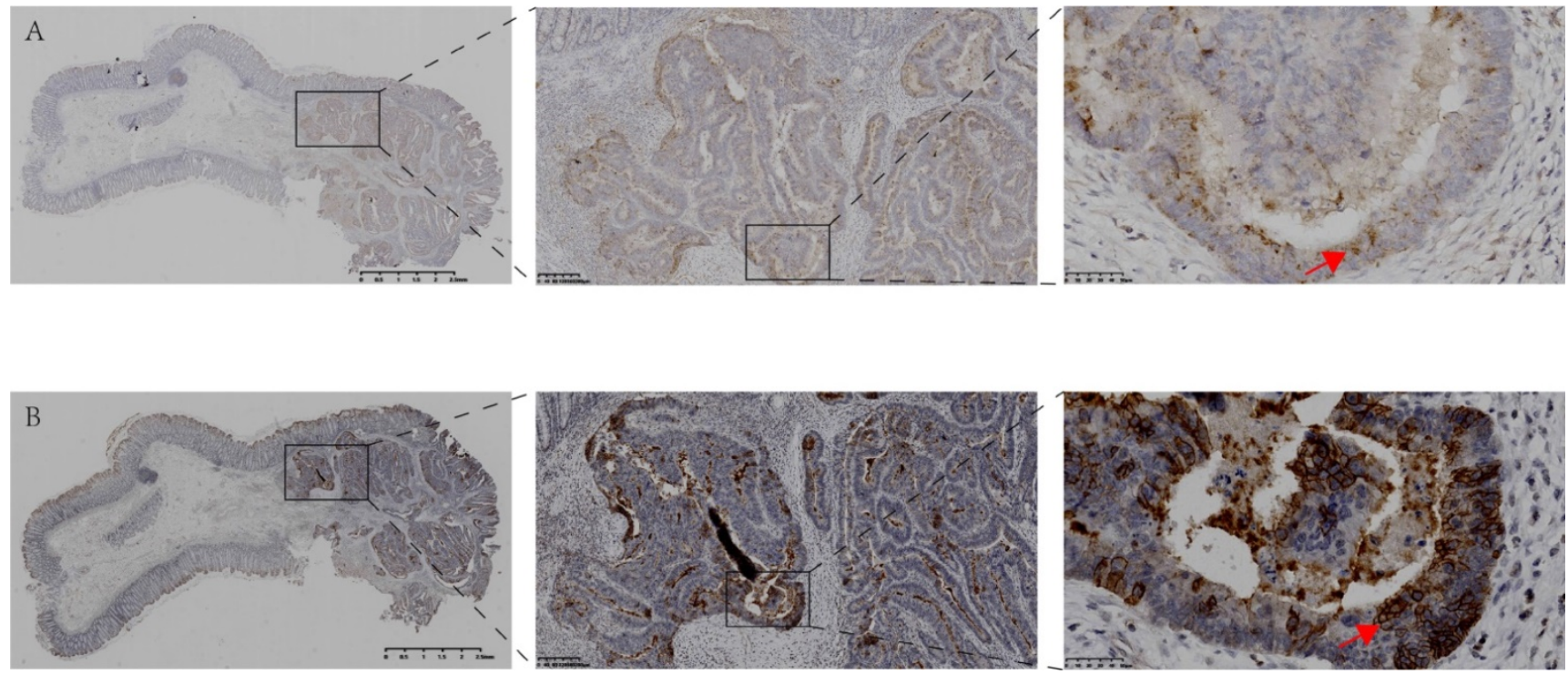

Figure 1. Expression and localization of CSF-1 and CSF-1R in the same human colon tissue. A. Immunohistochemical staining showed the expression and localization of CSF-1 in human colon cancer and para-cancerous tissues. B. Immunohistochemical staining demonstrated the expression and localization of CSF-1R in the same case. The expression of CSF-1 and CSF-1R in tumor tissue with abnormal structure was significantly higher than that in para-cancerous colon tissue with normal glandular structure. The expression level of CSF-1 in cancer cells is lower than that in tumor stroma, indicating TME may be the main sources of CSF1 (red arrow in Fig 1.A). CSF1-R is mainly expressed in tumor cell membrane (red arrow in Fig 1.B), which is different from the location of CSF-1. 
patients. Importantly, the authors provided evidence that tumor-associated macrophages-derived CXCL8 determines immune evasion through autonomous PD-L1 expression in gastric cancer, suggesting that it may be a promising strategy to block the CXCL8 pathway to increase anti-tumor immunity in gastric cancer [29]. The CSF-1/CSF-1R axis may be a biomarker for the clinical diagnosis of lymph node and peritoneal metastasis of gastric cancer and is a potential therapeutic target for gastric cancer[16]. Recombinant human granulocyte colony-stimulating factor (rhGCSF) has been widely used in the treatment of granulocytopenia induced by chemoradiotherapy. In vitro studies have shown that CSF can also be produced by tumor cells and stromal cells, and it could promote tumor fitness and cell proliferation and metastasis [67]. Patients with high CSF expression have a rapid disease progression and short survival time. Inhibition of CSF can reduce tumor angiogenesis and inhibit tumor growth. Fan et al. [68] studied the value of serum CSF in the diagnosis and prognosis of gastric cancer. It was found that the serum CSF level in gastric cancer patients was significantly higher than that in healthy patients. With advanced TNM stage, the serum level of CSF gradually decreased. High expression of CSF in gastric cancer tissues was significantly increased, which was positively correlated with TNM stage and lymph node metastasis [68, 69]. Morris et al [70] studied the expression and function of G-CSF and G-CSFR in gastrointestinal cancer tissues and cells. It was found that CSFR was highly expressed in 90\% of gastrointestinal cancer. Production of CSF was increased in interstitial myofibroblasts and cancer cells, and the proliferation and migration of cancer cells expressing CSF were enhanced. These processes depend on phosphorylation of the ERK1/RSK signaling pathway [70]. Scholars have found that CXCL8 is a key chemokine for gastric cancer metastasis, which is mainly derived from TAM. In gastric cancer, through inducing macrophages and PD-L1 to participate in the immunosuppression of the tumor microenvironment, CXCL8 inhibitors could trigger the anti-tumor response, which could provide potential therapeutic effects for gastric cancer patients [29]. Another research team studied the CSF3/CSF3R signaling in colon and rectal cancers, and results indicated that CSF3/CSF3R expression was correlated with changes in $\mathrm{T}$ cell and macrophage signatures and also correlated with genes that are associated with poor colorectal cancer prognosis [71].

Recent works showed that serum CSF-1 has great value in the diagnosis and progression of colorectal cancer (CRC), making it an independent prognostic factor for the survival of patients with CRC
[72]. As mentioned before, increased CSF-1R, CSF-1 and IL-34 expression in primary CRC was associated with a mesenchymal-like subtype and tumor invasion as well as distant metastasis [24, 72]. Recently, immunotherapy has gradually become the focus of cancer treatment. But most patients with "cold" tumors do not benefit from immunotherapy [63]. Interestingly, a targeted delivery strategy, which modified cell-penetrating TAT peptide by using CSF-1R inhibitor, successfully activate immune response through blocking the CSF-1/CSF-1R pathway and reducing M2 macrophages and thus promoting antitumor effector $\mathrm{CD}^{+} \mathrm{T}$-lymphocyte infiltration in "cold" colon cancer [64].

It has been found that abnormal expression of CSF-1/CSF-1R axis can also occur in malignant meningiomas, hepatocellular carcinoma, and pancreatic cancer, where CSF-1/CSF-1R blockade reprograms tumor-infiltrating macrophages and improves response improves response to $\mathrm{T}$ cell checkpoint immunotherapy [66, 73, 74].

The tumor microenvironment includes the structure, function and metabolism of the tissues in which tumors are located, as well as the external environment in which tumors themselves and surrounding immune cells and immunoregulatory factors are formed [75]. The occurrence, growth and metastasis of tumors are closely related to the microenvironment. CSF can recruit neutrophils, monocytes and macrophages, etc. to the adjacent areas of the tumors, promoting the development of tumors. Li et al. found that the infiltration of bone marrow-derived suppressor cells and macrophages in a mouse colorectal cancer model was related to the increase of CSF. CSF could mobilize bone marrow-derived suppressor cells to migrate from bone marrow to the colon, promoting the proliferation of bone marrow-derived suppressor cells and inhibiting their apoptosis and then inducing the occurrence and development of colon cancer [76]. Studies have reported [77] that TAM in GI tumor microenvironment, responding to stimuli such as growth factors and cytokines, could be polarized into a state with pro-tumor activity or anti-tumor activity (M1 or M2). CSF is a cytokine that can affect immune cells in the tumor microenvironment and has tumorpromoting activity (Fig. 2). A study investigated the effect of CSF/CSF-R on the progression of colon cancer and pancreatic cancer in a mouse model and the results showed that in the absence of CSF-R, macrophage-related tumor cytotoxicity was amplified, indicating that CSF/CSF-R is an important clinical application target for controlling tumor microenvironment and gastrointestinal tumor progression $[72,78]$. 


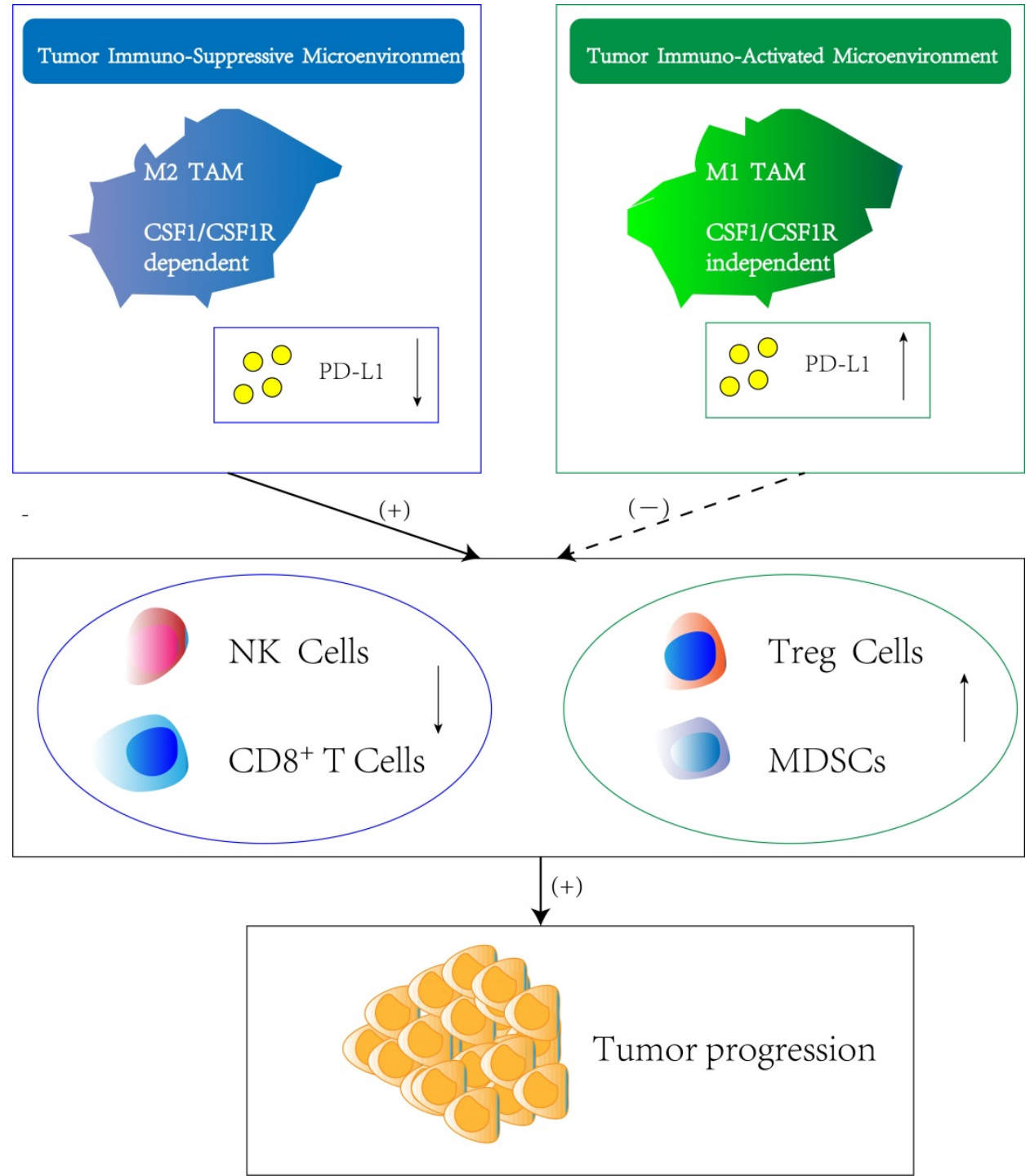

Figure 2. Regulation of immune suppression or activation by TAM subtypes. Macrophage polarization within the tumor immune-suppressive microenvironment is highly dependent on CSF-1/CSF-1R axis which originates either from tumor cells or stromal cells. The M2 TAM phenotype induce the downregulation of PD-L1 in TME, which result in silencing of immune effector cells such as nature killing cells and CD8+ $T$ cells. Meanwhile, the infiltration and function of other immune-suppressive cell types such as $T$ regulatory cells (Treg cells) and Myeloid-derived suppressor cells (MDSCs) is stimulated, thus promoting tumor progression. In contrast, MI TAM are attributed with tumoricidal functions showing the opposite effect of M2 TAM.

\section{Signaling pathway regulation mechanisms involved by CSF-1 and CSF-1R}

CSF-1R binding to the CSF-1 ligand on the surface of cancer cells can activate multiple intracellular signaling pathways to promote proliferation, invasion, metastasis, and angiogenesis [79]. Blocking CSF-1R with antibodies can reduce the activation of CSF-1 and prevent the proliferation and metastasis of malignant tumors. It was found that CSF could participate in the activation of the JAK tyrosine kinase pathway [80], the Wnt3a pathway [81], the PI3-kinase pathway [82], and the ERK1/2 pathway [83] (Fig. 3). JAK/STAT is generally believed to be the main pathway for CSF signal transmission [80]. After CSF binds to its receptor, JNK is activated, which could phosphorylate tyrosine residues in the intracellular segment of CSFR and then re-localize the STAT family proteins into the nucleus, binding to the promoter region of the target gene and inducing effector protein expression [84]. Recent work has demonstrated that the elevated expression of receptor tyrosine kinase CSF-1R act as a direct miR-34a target and as a negative effector of $\mathrm{p} 53 / \mathrm{miR}-34 \mathrm{a}$ axis, promoting progression of colorectal cancer by activating STAT3 pathway [24]. Notably, CSF-1R does not necessarily receive the stimulation of CSF-1. For example, by triggering receptor expressed on myoid cells-2 (TREM2), CSF-1R is a high risk marker for Alzheimer's disease, which explains the disadvantage of mono targeting CSF-1R from another point of view [85]. 


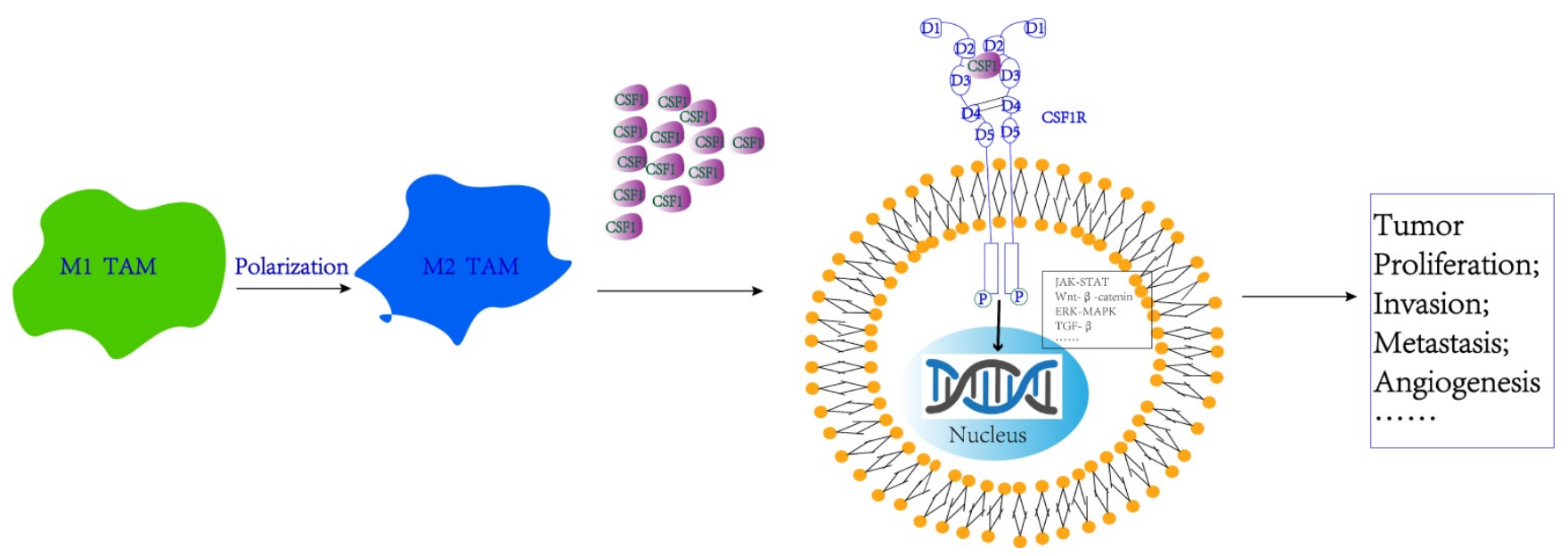

Figure 3. Signaling pathway regulation mechanisms involved by CSF-1 and CSF-1R. CSF-1R binding to the CSF-1 ligand on the surface of cancer cells can activate multiple intracellular signaling pathways, including JAK-STAT, Wnt, MAPK, TGF- $\beta$ signaling pathway, thus promoting tumor proliferation, invasion, metastasis, and angiogenesis.

Understanding the signaling mechanism of CSF-1 and CSF-1R in cancer and other diseases and taking appropriate measures to block CSF-1/CSF-1R signaling is a promising new immunotherapy with potential for future clinical application. Studies have found that CSF-1 is one of the most common proinflammatory cytokines, and it can cause various inflammatory diseases [65]. It plays an important role in the development and progression of osteoarthritis, cancer and other autoimmune diseases. CSF-1 plays a role by binding CSF-1R, causing a cascade reaction of signaling pathways leading to cell proliferation and differentiation, promoting the differentiation and survival of monocytes, macrophages and osteoclasts. CSF-1R is overexpressed in many cancers and cancer-related macrophages and is therefore used as a drug target for cancer and inflammatory diseases. Some CSF-1R inhibitors have been successfully applied to these diseases.

\section{Summary}

CSF is widely used in clinic to treat granulocytopenia patients after chemotherapy. Increasing research has shown that CSF-1 and CSF-1R are expressed in malignant tumors of the digestive tract and can promote the growth, migration and invasion of tumors. CSF-1 and CSF-1R play a role through multiple signaling pathways by inhibiting the immune response of the body. Blocking the CSF-1/CSF-1R axis can not only directly treat tumor cells with CSF1R, as a targeted therapy, but also can promote the polarization of TAM and improve the tumor microenvironment, thereby exerting an anti-tumor effect. However, the mechanism of CSF-1 and CSF-1R in GI malignant tumors is still not clear, and the mechanism of how inflammatory immune cells participate in the occurrence and development of malignant tumors is not perfectly defined. Therefore, more in-depth exploration and clinical validation are needed to improve the effects of targeted drugs and comprehensive treatments and to help identify new markers of malignant tumors for early diagnosis and evaluation of prognosis.

\section{Acknowledgements}

This study was supported by the Education Foundation of Liaoning Province (Grant number: JC2019021).

\section{Competing Interests}

The authors have declared that no competing interest exists.

\section{References}

1. Torpy JM, Lynm C, Glass RM. JAMA patient page. Stomach cancer. JAMA. 2010; 303: 1771.

2. Jemal A, Center MM, DeSantis C, Ward EM. Global patterns of cancer incidence and mortality rates and trends. Cancer Epidemiol Biomarkers Prev. 2010; 19: 1893-907.

3. Blay JY, Kang YK, Nishida T, von Mehren M. Gastrointestinal stromal tumours. Nat Rev Dis Primers. 2021; 7: 22.

4. Sundar R, Liu DH, Hutchins GG, Slaney HL, Silva AN, Oosting J, et al. Spatial profiling of gastric cancer patient-matched primary and locoregional metastases reveals principles of tumour dissemination. Gut. 2020; [Epub ahead of print].

5. Schmidt DR, Patel R, Kirsch DG, Lewis CA, Vander Heiden MG, Locasale JW. Metabolomics in cancer research and emerging applications in clinical oncology. CA Cancer J Clin. 2021; 71: 333-58

6. Nagaoka K, Shirai M, Taniguchi K, Hosoi A, Sun C, Kobayashi Y, et al. Deep immunophenotyping at the single-cell level identifies a combination of anti-IL-17 and checkpoint blockade as an effective treatment in a preclinical model of data-guided personalized immunotherapy. Journal for immunotherapy of cancer. 2020; 8(2): e001358.

7. Karagiannidis I, Jerman SJ, Jacenik D, Phinney BB, Yao R, Prossnitz ER, et al. G-CSF and G-CSFR Modulate CD4 and CD8 T Cell Responses to Promote Colon Tumor Growth and Are Potential Therapeutic Targets. Frontiers in immunology. 2020; 11: 1885.

8. Liongue C, Wright C, Russell AP, Ward AC. Granulocyte colony-stimulating factor receptor: stimulating granulopoiesis and much more. Int J Biochem Cell Biol. 2009; 41: 2372-5.

9. Touw IP, van de Geijn GJ. Granulocyte colony-stimulating factor and its receptor in normal myeloid cell development, leukemia and related blood cell disorders. Front Biosci. 2007; 12: 800-15.

10. Wright CR, Ward AC, Russell AP. Granulocyte Colony-Stimulating Factor and Its Potential Application for Skeletal Muscle Repair and Regeneration. Mediators Inflamm. 2017; 2017: 7517350. 
11. Fujiwara T, Yakoub MA, Chandler A, Christ AB, Yang G, Ouerfelli O, et al. CSF1/CSF1R Signaling Inhibitor Pexidartinib (PLX3397) Reprograms TumorAssociated Macrophages and Stimulates T-cell Infiltration in the Sarcoma Microenvironment. Molecular cancer therapeutics. 2021; 20(8): 1388-99.

12. Quail DF, Bowman RL, Akkari L, Quick ML, Schuhmacher AJ, Huse JT, et al. The tumor microenvironment underlies acquired resistance to CSF-1R inhibition in gliomas. Science (New York, NY). 2016; 352: aad3018.

13. Ao JY, Zhu XD, Chai ZT, Cai H, Zhang YY, Zhang KZ, et al. ColonyStimulating Factor 1 Receptor Blockade Inhibits Tumor Growth by Altering the Polarization of Tumor-Associated Macrophages in Hepatocellular Carcinoma. Molecular cancer therapeutics. 2017; 16: 1544-54.

14. Hsu WC, Lee YC, Liang PI, Chang LL, Huang AM, Lin HH, et al. CSF-1 Overexpression Predicts Poor Prognosis in Upper Tract Urothelial Carcinomas. Disease markers. 2019; 2019: 2724948.

15. Li KY, Yuan JL, Trafton D, Wang JX, Niu N, Yuan CH, et al. Pancreatic ductal adenocarcinoma immune microenvironment and immunotherapy prospects. Chronic diseases and translational medicine. 2020; 6: 6-17.

16. Okugawa Y, Toiyama Y, Ichikawa T, Kawamura M, Yasuda H, Fujikawa H, et al. Colony-stimulating factor-1 and colony-stimulating factor-1 receptor co-expression is associated with disease progression in gastric cancer. Int J Oncol. 2018; 53: 737-49.

17. Yuan X, Li Y, Zhang AZ, Jiang CH, Li FP, Xie YF, et al. Tumor-associated macrophage polarization promotes the progression of esophageal carcinoma. Aging (Albany NY). 2020; 13: 2049-72.

18. Cannarile MA, Weisser $\mathrm{M}$, Jacob W, Jegg AM, Ries $\mathrm{CH}$, Ruttinger D. Colony-stimulating factor 1 receptor (CSF1R) inhibitors in cancer therapy. J Immunother Cancer. 2017; 5: 53.

19. Li M, Li M, Yang Y, Liu Y, Xie H, Yu Q, et al. Remodeling tumor immune microenvironment via targeted blockade of PI3K- $\gamma$ and CSF-1/CSF-1R pathways in tumor associated macrophages for pancreatic cancer therapy. J Control Release. 2020; 321: 23-35.

20. Tan IL, Arifa RDN, Rallapalli H, Kana V, Lao Z, Sanghrajka RM, et al. CSF1R inhibition depletes tumor-associated macrophages and attenuates tumor progression in a mouse sonic Hedgehog-Medulloblastoma model. Oncogene. 2021; 40: 396-407.

21. Sauter KA, Waddell LA, Lisowski ZM, Young R, Lefevre L, Davis GM, et al. Macrophage colony-stimulating factor (CSF1) controls monocyte production and maturation and the steady-state size of the liver in pigs. Am J Physiol Gastrointest Liver Physiol. 2016; 311: G533-47.

22. Ensan S, Li A, Besla R, Degousee N, Cosme J, Roufaiel M, et al. Self-renewing resident arterial macrophages arise from embryonic CX3CR1(+) precursors and circulating monocytes immediately after birth. Nat Immunol. 2016; 17: $159-68$

23. Chambers SK, Kacinski BM, Ivins CM, Carcangiu ML. Overexpression of epithelial macrophage colony-stimulating factor (CSF-1) and CSF-1 receptor: a poor prognostic factor in epithelial ovarian cancer, contrasted with a protective effect of stromal CSF-1. Clin Cancer Res. 1997; 3: 999-1007.

24. Shi $\mathrm{X}$, Kaller $\mathrm{M}$, Rokavec $\mathrm{M}$, Kirchner $\mathrm{T}$, Horst $\mathrm{D}$, Hermeking $\mathrm{H}$. Characterization of a p53/miR-34a/CSF1R/STAT3 Feedback Loop in Colorectal Cancer. Cellular and molecular gastroenterology and hepatology. 2020; 10: 391-418.

25. Fontana MF, de Melo GL, Anidi C, Hamburger R, Kim CY, Lee SY, et al. Macrophage Colony Stimulating Factor Derived from CD4+ T Cells Contributes to Control of a Blood-Borne Infection. PLoS Pathog. 2016; 12: e1006046.

26. Gu H, Wang B, He J, Hu Y. Macrophage colony stimulating factor (MCSF) of Japanese flounder (Paralichthys olivaceus): Immunoregulatory property, anti-infectious function, and interaction with MCSF receptor. Developmental and comparative immunology. 2021; 116: 103920.

27. Deng P, Wang YL, Pattengale PK, Rettenmier CW. The role of individual cysteine residues in the processing, structure, and function of human macrophage colony-stimulating factor. Biochemical and biophysical research communications. 1996; 228: 557-66.

28. Hanington PC, Wang T, Secombes CJ, Belosevic M. Growth factors of lower vertebrates: characterization of goldfish (Carassius auratus L.) macrophage colony-stimulating factor-1. The Journal of biological chemistry. 2007; 282: 31865-72.

29. Lin $\mathrm{C}, \mathrm{He} \mathrm{H}$, Liu $\mathrm{H}, \mathrm{Li} \mathrm{R}$, Chen $\mathrm{Y}, \mathrm{Qi} \mathrm{Y}$, et al. Tumour-associated macrophages-derived CXCL8 determines immune evasion through autonomous PD-L1 expression in gastric cancer. Gut. 2019; 68: 1764-73.

30. Tacke F, Wynn TA. Biomarker and Therapeutic Potential of CSF1 in Acute Liver Failure. Gastroenterology. 2015; 149: 1675-8.

31. Murga-Zamalloa C, Rolland DCM, Polk A, Wolfe A, Dewar H, Chowdhury P, et al. Colony-Stimulating Factor 1 Receptor (CSF1R) Activates AKT/mTOR Signaling and Promotes T-Cell Lymphoma Viability. Clinical cancer research : an official journal of the American Association for Cancer Research. 2020; 26: 690-703

32. El-Gamal MI, Al-Ameen SK, Al-Koumi DM, Hamad MG, Jalal NA, Oh CH. Recent Advances of Colony-Stimulating Factor-1 Receptor (CSF-1R) Kinase and Its Inhibitors. Journal of medicinal chemistry. 2018; 61: 5450-66.

33. Sherr CJ. The fms oncogene. Biochim Biophys Acta. 1988; 948: 225-43.

34. Wang Y, Yeung YG, Stanley ER. CSF-1 stimulated multiubiquitination of the CSF-1 receptor and of $\mathrm{Cbl}$ follows their tyrosine phosphorylation and association with other signaling proteins. Journal of cellular biochemistry. 1999; 72: 119-34.
35. Hamilton JA. CSF-1 signal transduction. J Leukoc Biol. 1997; 62: 145-55.

36. Lou J, Low-Nam ST, Kerkvliet JG, Hoppe AD. Delivery of CSF-1R to the lumen of macropinosomes promotes its destruction in macrophages. Journal of cell science. 2014; 127: 5228-39.

37. Choudhury GG, Sylvia VL, Pfeifer A, Wang LM, Smith EA, Sakaguchi AY. Human colony stimulating factor-1 receptor activates the C-raf-1 proto-oncogene kinase. Biochem Biophys Res Commun. 1990; 172: 154-9.

38. Song Z, Wang M, Ge Y, Chen XP, Xu Z, Sun Y, et al. Tyrosine phosphatase SHP2 inhibitors in tumor-targeted therapies. Acta pharmaceutica Sinica B. 2021; 11: 13-29.

39. Pollard JW. Role of colony-stimulating factor-1 in reproduction and development. Mol Reprod Dev. 1997; 46: 54-60; discussion -1.

40. Saleh R, Lee MC, Khiew SH, Louis C, Fleetwood AJ, Achuthan A, et al. CSF-1 in Inflammatory and Arthritic Pain Development. Journal of immunology (Baltimore, Md : 1950). 2018; 201: 2042-53.

41. Laoui D, Van Overmeire E, De Baetselier P, Van Ginderachter JA, Raes G. Functional Relationship between Tumor-Associated Macrophages and Macrophage Colony-Stimulating Factor as Contributors to Cancer Progression. Frontiers in immunology. 2014; 5: 489.

42. Wang Y, Tiruthani K, Li S, Hu M, Zhong G, Tang Y, et al. mRNA Delivery of a Bispecific Single-Domain Antibody to Polarize Tumor-Associated Macrophages and Synergize Immunotherapy against Liver Malignancies. Advanced materials (Deerfield Beach, Fla). 2021; 33: e2007603.

43. Wenes M, Shang M, Di Matteo M, Goveia J, Martín-Pérez R, Serneels J, et al. Macrophage Metabolism Controls Tumor Blood Vessel Morphogenesis and Metastasis. Cell metabolism. 2016; 24: 701-15.

44. Chen YC, Lai YS, Hsuuw YD, Chang KT. Withholding of M-CSF Supplement Reprograms Macrophages to M2-Like via Endogenous CSF-1 Activation. International journal of molecular sciences. 2021; 22(7): 3532.

45. Ries $\mathrm{CH}$, Cannarile MA, Hoves S, Benz J, Wartha K, Runza V, et al. Targeting tumor-associated macrophages with anti-CSF-1R antibody reveals a strategy for cancer therapy. Cancer cell. 2014; 25: 846-59.

46. Pyonteck SM, Akkari L, Schuhmacher AJ, Bowman RL, Sevenich L, Quail DF, et al. CSF-1R inhibition alters macrophage polarization and blocks glioma progression. Nature medicine. 2013; 19: 1264-72.

47. Jin X, Su H, Xu L, Wang Y, Su R, Zhang Z, et al. Different co-culture models reveal the pivotal role of TBBPA-promoted M2 macrophage polarization in the deterioration of endometrial cancer. J Hazard Mater. 2021; 413: 125337.

48. Hua F, Tian Y, Gao Y, Li C, Liu X. Colony-stimulating factor 1 receptor inhibition blocks macrophage infiltration and endometrial cancer cell proliferation. Molecular medicine reports. 2019; 19: 3139-47.

49. Xia Y, Rao L, Yao H, Wang Z, Ning P, Chen X. Engineering Macrophages for Cancer Immunotherapy and Drug Delivery. Advanced materials (Deerfield Beach, Fla). 2020; 32: e2002054.

50. Vitale I, Manic G, Coussens LM, Kroemer G, Galluzzi L. Macrophages and Metabolism in the Tumor Microenvironment. Cell metabolism. 2019; 30: 36-50.

51. Chen Y, Song Y, Du W, Gong L, Chang H, Zou Z. Tumor-associated macrophages: an accomplice in solid tumor progression. Journal of biomedical science. 2019; 26: 78

52. Autio KA, Klebanoff CA, Schaer D, Kauh JSW, Slovin SF, Adamow M, et al. Immunomodulatory Activity of a Colony-stimulating Factor-1 Receptor Inhibitor in Patients with Advanced Refractory Breast or Prostate Cancer: A Phase I Study. Clinical cancer research : an official journal of the American Association for Cancer Research. 2020; 26: 5609-20.

53. Adamopoulos IE, Mellins ED. Alternative pathways of osteoclastogenesis in inflammatory arthritis. Nature reviews Rheumatology. 2015; 11: 189-94.

54. Benner B, Good L, Quiroga D, Schultz TE, Kassem M, Carson WE, et al. Pexidartinib, a Novel Small Molecule CSF-1R Inhibitor in Use for Tenosynovial Giant Cell Tumor: A Systematic Review of Pre-Clinical and Clinical Development. Drug design, development and therapy. 2020; 14: 1693-704.

55. Lin W, Xu D, Austin CD, Caplazi P, Senger K, Sun Y, et al. Function of CSF1 and IL34 in Macrophage Homeostasis, Inflammation, and Cancer. Frontiers in immunology. 2019; 10: 2019.

56. Wei S, Nandi S, Chitu V, Yeung YG, Yu W, Huang M, et al. Functional overlap but differential expression of CSF-1 and IL-34 in their CSF-1 receptor-mediated regulation of myeloid cells. Journal of leukocyte biology. 2010; 88: 495-505.

57. Razak AR, Cleary JM, Moreno V, Boyer M, Calvo Aller E, Edenfield W, et al. Safety and efficacy of AMG 820, an anti-colony-stimulating factor 1 receptor antibody, in combination with pembrolizumab in adults with advanced solid tumors. Journal for immunotherapy of cancer. 2020; 8(2): e001006.

58. Magkouta SF, Vaitsi PC, Pappas AG, Iliopoulou M, Kosti CN, Psarra K, et al. CSF1/CSF1R Axis Blockade Limits Mesothelioma and Enhances Efficiency of Anti-PDL1 Immunotherapy. Cancers. 2021; 13(11): 2546.

59. Wang Y, Szretter KJ, Vermi W, Gilfillan S, Rossini C, Cella M, et al. IL-34 is a tissue-restricted ligand of CSF1R required for the development of Langerhans cells and microglia. Nature immunology. 2012; 13: 753-60.

60. Buechler $\mathrm{MB}, \mathrm{Fu} \mathrm{W}$, Turley SJ. Fibroblast-macrophage reciprocal interactions in health, fibrosis, and cancer. Immunity. 2021; 54: 903-15.

61. Quaranta V, Rainer C, Nielsen SR, Raymant ML, Ahmed MS, Engle DD, et al. Macrophage-Derived Granulin Drives Resistance to Immune Checkpoint Inhibition in Metastatic Pancreatic Cancer. Cancer research. 2018; 78: 4253-69.

62. Czako B, Marszalek JR, Burke JP, Mandal P, Leonard PG, Cross JB, et al. Discovery of IACS-9439, a Potent, Exquisitely Selective, and Orally 
Bioavailable Inhibitor of CSF1R. Journal of medicinal chemistry. 2020; 63: 9888-911.

63. Duan $\mathrm{Q}$, Zhang H, Zheng J, Zhang L. Turning Cold into Hot: Firing up the Tumor Microenvironment. Trends in cancer. 2020; 6: 605-18.

64. Fang $\mathrm{Y}, \mathrm{He} \mathrm{Y}, \mathrm{Wu} \mathrm{C}, \mathrm{Zhang} \mathrm{M}, \mathrm{Gu} \mathrm{Z}$, Zhang J, et al. Magnetism-mediated targeting hyperthermia-immunotherapy in "cold" tumor with CSF1R inhibitor. Theranostics. 2021; 11: 6860-72.

65. Kumari A, Silakari O, Singh RK. Recent advances in colony stimulating factor-1 receptor/c-FMS as an emerging target for various therapeutic implications. Biomed Pharmacother. 2018; 103: 662-79.

66. Zhu Y, Knolhoff BL, Meyer MA, Nywening TM, West BL, Luo J, et al. CSF1/CSF1R blockade reprograms tumor-infiltrating macrophages and improves response to T-cell checkpoint immunotherapy in pancreatic cancer models. Cancer research. 2014; 74: 5057-69.

67. Cho H, Kim JH, Jun CD, Jung DW, Williams DR. CAF-Derived IL6 and GM-CSF Cooperate to Induce M2-like TAMs-Response. Clinical cancer research : an official journal of the American Association for Cancer Research. 2019; 25: 894-5.

68. Fan Z, Li Y, Zhao Q, Fan L, Tan B, Zuo J, et al. Highly Expressed Granulocyte Colony-Stimulating Factor (G-CSF) and Granulocyte Colony-Stimulating Factor Receptor (G-CSFR) in Human Gastric Cancer Leads to Poor Survival. Med Sci Monit. 2018; 24: 1701-11.

69. Zhang $X, X u$ W. Neutrophils diminish T-cell immunity to foster gastric cancer progression: the role of GM-CSF/PD-L1/PD-1 signalling pathway. Gut. 2017; 66: 1878-80.

70. Morris KT, Khan H, Ahmad A, Weston LL, Nofchissey RA, Pinchuk IV, et al. G-CSF and G-CSFR are highly expressed in human gastric and colon cancers and promote carcinoma cell proliferation and migration. British journal of cancer. 2014; 110: 1211-20.

71. Saunders AS, Bender DE, Ray AL, Wu X, Morris KT. Colony-stimulating factor 3 signaling in colon and rectal cancers: Immune response and CMS classification in TCGA data. PLoS One. 2021; 16: e0247233.

72. Łukaszewicz-Zając M, Mroczko B. Circulating Biomarkers of Colorectal Cancer (CRC)-Their Utility in Diagnosis and Prognosis. Journal of clinical medicine. 2021; 10(11): 2391.

73. Yeung J, Yaghoobi V, Miyagishima D, Vesely MD, Zhang T, Badri T, et al. Targeting the CSF1/CSF1R Axis is a Potential Treatment Strategy for Malignant Meningiomas. Neuro-oncology. 2021; Epub ahead of print].

74. Zhu Y, Yang J, Xu D, Gao XM, Zhang Z, Hsu JL, et al. Disruption of tumour-associated macrophage trafficking by the osteopontin-induced colony-stimulating factor-1 signalling sensitises hepatocellular carcinoma to anti-PD-L1 blockade. Gut. 2019; 68: 1653-66.

75. Kaymak I, Williams KS, Cantor JR, Jones RG. Immunometabolic Interplay in the Tumor Microenvironment. Cancer cell. 2021; 39: 28-37.

76. Li W, Zhang X, Chen Y, Xie Y, Liu J, Feng Q, et al. G-CSF is a key modulator of MDSC and could be a potential therapeutic target in colitis-associated colorectal cancers. Protein Cell. 2016; 7: 130-40.

77. Karagiannidis I, de Santana Van Vilet E, Said Abu Egal E, Phinney B, Jacenik D, Prossnitz ER, et al. G-CSF and G-CSFR Induce a Pro-Tumorigenic Macrophage Phenotype to Promote Colon and Pancreas Tumor Growth. Cancers. 2020; 12(10): 2868.

78. Metcalf $\mathrm{D}$. The colony-stimulating factors and cancer. Nature reviews Cancer. 2010; 10: 425-34

79. Sun $\mathrm{L}$, Liang $\mathrm{H}, \mathrm{Yu} \mathrm{W}$, Jin $\mathrm{X}$. Increased invasive phenotype of CSF-1R expression in glioma cells via the ERK1/2 signaling pathway. Cancer gene therapy. 2019; 26: 136-44.

80. Fujita Y, Matsuoka N, Temmoku J, Furuya-Yashiro M, Asano T, Sato S, et al. JAK inhibitors impair GM-CSF-mediated signaling in innate immune cells. BMC immunology. 2020; 21: 35.

81. Fujita K, Janz S. Attenuation of WNT signaling by DKK-1 and -2 regulates BMP2-induced osteoblast differentiation and expression of OPG, RANKL and M-CSF. Molecular cancer. 2007: 6: 71.

82. Dwyer AR, Greenland EL, Pixley FJ. Promotion of Tumor Invasion by TumorAssociated Macrophages: The Role of CSF-1-Activated Phosphatidylinositol 3 Kinase and Src Family Kinase Motility Signaling. Cancers (Basel). 2017; 9(6): 68.

83. Ding J, Yang C, Zhang Y, Wang J, Zhang S, Guo D, et al. M2 macrophagederived G-CSF promotes trophoblasts EMT, invasion and migration via activating PI3K/Akt/Erk1/2 pathway to mediate normal pregnancy. Journal of cellular and molecular medicine. 2021; 25: 2136-47.

84. Wang Y, Cai D, Brendel C, Barett C, Erben P, Manley PW, et al. Adaptive secretion of granulocyte-macrophage colony-stimulating factor (GM-CSF) mediates imatinib and nilotinib resistance in $\mathrm{BCR} / \mathrm{ABL}+$ progenitors via JAK-2/STAT-5 pathway activation. Blood. 2007; 109: 2147-55.

85. Cheng B, Li X, Dai K, Duan S, Rong Z, Chen Y, et al. Triggering Receptor Expressed on Myeloid Cells-2 (TREM2) Interacts With Colony-Stimulating Factor 1 Receptor (CSF1R) but Is Not Necessary for CSF1/CSF1R-Mediated Microglial Survival. Frontiers in immunology. 2021; 12: 633796. 